\title{
AN EFFICIENT POWER CONTROL GAME MODEL FOR WIRELESS SENSOR NETWORKS
}

\author{
O. I. Ladipo ${ }^{1, *}$ and A. 0 Gbenga-Ilori ${ }^{2}$

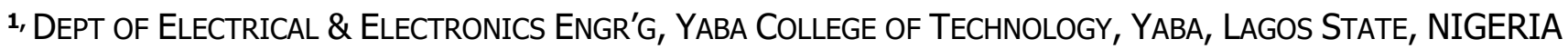 \\ 2, DEPT OF ElECTRICAL \& ELECTRONICS ENGR'G, UNIVERSITY OF LAGOS, AKOKA- LAGOS, LAGOS STATE, NIGERIA \\ E-mail addresses: ${ }^{1}$ oluwabusayo.ladipo@yabatech.edu.ng, ${ }^{2}$ gbengailori@unilag.edu.ng
}

\begin{abstract}
Efficient energy usage is a major design challenge in wireless sensor networks. In this paper, an efficient power control scheme that mitigates interference and reduces the energy usage of the sensor nodes in a wireless sensor network is presented using the game theory. A non-cooperative game was formulated among the sensor nodes in the modeled network by setting a transmission power limit at the receiving nodes which ensured that the transmitting nodes transmits at the optimal power level. The utility of the sensor nodes and the interference proportion within the network was evaluated at the optimal and discrete transmit powers. The Nash equilibrium of the proposed game was studied and it corresponds to a stability point where the network performance was optimized. Simulation results showed that the proposed scheme is effective for optimization of network resource utilization, reduction in the energy consumption of the nodes, increasing the transmission sum rate, reduction of interference within the network, and improving the network capacity.
\end{abstract}

Keywords: Power control, Wireless sensor network, Non-cooperative game, Interference, Energy.

\section{INTRODUCTION}

Several autonomous sensor nodes working together to detect, monitor, and determine diverse operations, events or parameters at different locations are referred to as wireless sensor network (WSN). This networks are less expensive to set up because they require less infrastructures, they are multi-functional, capable of carrying out in-network analysis, embedded with sensing mechanism, able to process information, and are mostly deployed far from human intervention $[1,2]$. Effective power control is a major limitation in the performance of this network because all the operations of the sensor nodes depend largely on their battery power and as a result, they use up their energy very fast [3]. A sensor node aims to transmit with high power in order to meet the required quality of service and to ensure successful packet delivery. This high transmit power increases the level of interference caused to the neighboring nodes which results in an increase in their transmit power. The effect of transmitting with high power causes jeopardy in the network and thus, it is of utmost importance that sensor nodes transmits at optimal transmit power to conserve their energy and to keep the interference proportion within the network at a minima/ level.

The nature of operation of WSN is different when compared with wireless heterogeneous network and wired network because the sensor nodes have restricted energy storage, communicate via short distances, lack global information, have varying network topology, and have limited computational capability [4-6]. These peculiarities in the operation of WSN has made the concept of game theory, which is an effective optimization technique, an important tool in analyzing interactive scenarios among decision makers in the design of WSN $[3,7,8]$. Game theory has been applied in several works of literature to propose different solution to the problem of power control in WSN and also in wireless cellular network as seen in [9-12]. A multi-source sensor network was

* Corresponding author, tel: +234- $803-483-5227$ 
presented in [13] where the sensor node aims to transmit with an optimal power to achieve a target signal to interference plus noise ratio (SINR) at the master sensor. The work in [13] resulted in distancedependent attenuation with various path loss exponent but the interference from the master node was not considered which is in contrast to our formulated power control game where the optimal power of all the sensor nodes was determined by considering all the resulting interference from all transmitting nodes.

In the work of [14], a power control algorithm with incomplete information was proposed and a Bayesian Nash equilibrium was used to determine the stability point in the proposed method. A price based distributed power control scheme was proposed for WSN in [15] while [16] and [17] focused on a restrictive energy distributed self-adaptive algorithm. The authors in these works aimed at reducing the energy consumption of the sensor nodes but the interference among the nodes during transmission was not considered. Motivated by these limitations, in this paper, we develop an energy-efficient power control scheme that mitigate interference between the sensor nodes, increases transmission sum rate, and improves the overall network capacity. The sensor nodes within the modeled network act as the players, their choice of transmitter power is their respective strategy and the utility of the nodes are measured in terms of transmission sum rate, reduced interference, and increased lifespan of the sensor nodes. A dynamic power threshold was set at each sensor nodes which ensures that all the transmitting nodes transmit at their optimal power and determined a utility function that incorporates both the transmission gain and the cost function which serves as a penalty for the nodes that transmit above the optimal power threshold. The sensor nodes update their power strategy as a result of this utility function and they aim to transmit at the optimal transmit power that maximizes their respective utility.

The major contributions of this work includes:

- Formulation of a non-cooperative power control game that reduces interference and energy consumption in a wireless sensor network by setting a power threshold at each sensor node.

- Proposal of an appropriate utility function that maximizes the degree of satisfaction of the sensor nodes which was defined in terms of increased capacity and transmission sum rate. A cost function which is a measure of the amount of interference the sensor nodes cause during their active transmission was incorporated into the utility function and it serves as a penalty for the nodes transmitting higher than the set threshold.

- Determination of the equilibrium point in the proposed scheme which is the stability point where the system performance is optimized.

The remainder of this paper are as follows: Section 2 presents the system model and assumptions of the power control game. Section 3 provides the illustrations of the proposed scheme and the proof of the existence of the Nash equilibrium. In section 4, the simulation results and technical discussions are presented while the conclusions of this paper are given in section 5 .

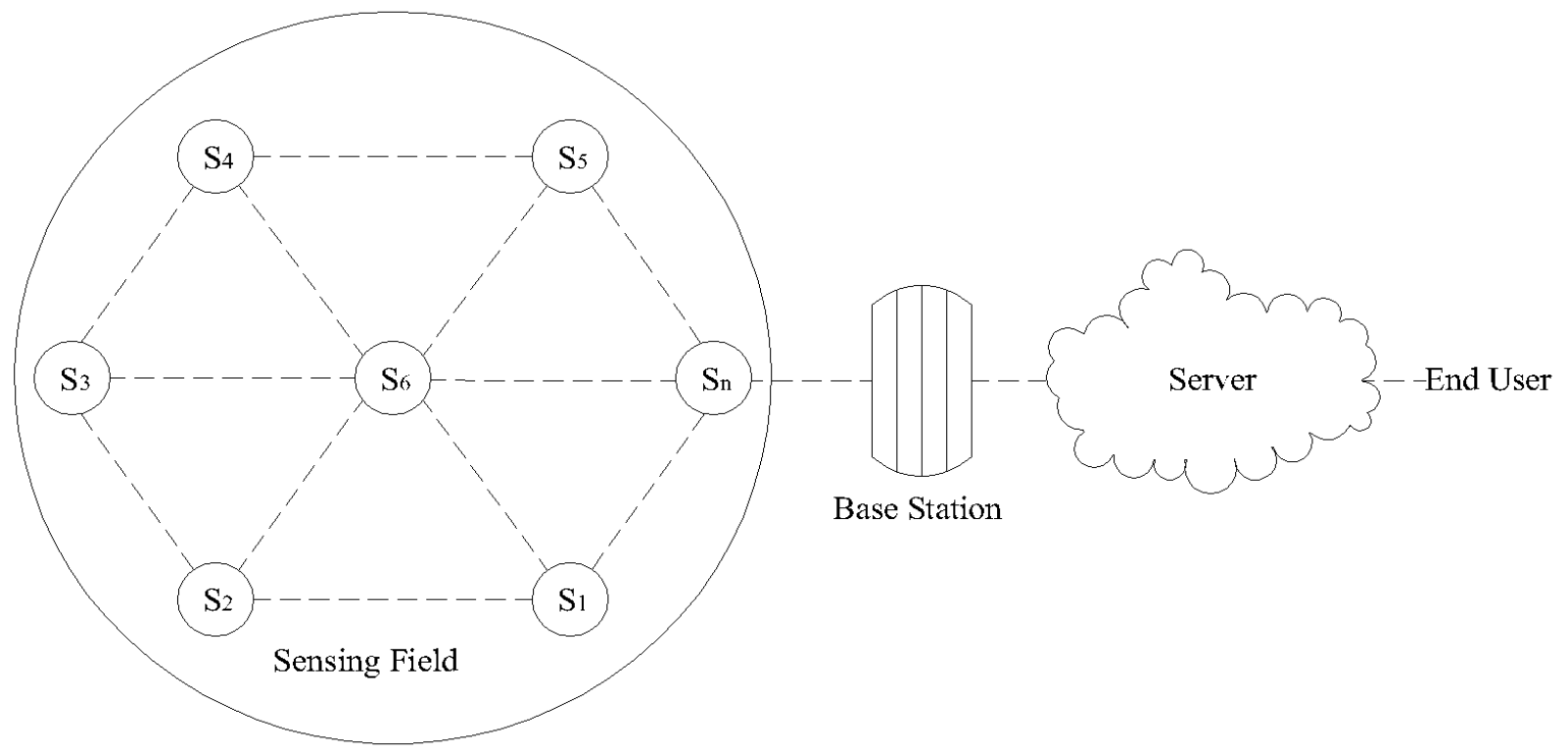

Figure 1: A system model of a wireless sensor network 


\section{SYSTEM MODEL}

A wireless sensor network that comprises of $S$ sensor nodes, a base station, and a server shown in Figure 1 is modeled in this paper. All the sensor nodes in the network act as the players, their transmission power is their set of strategy, and their utility is a measure of increased transmission sum rate, reduced interference, and efficient energy consumption. The sensor nodes are distributed in an arbitrary manner within the network and are represented by set $S=$ $\left\{S_{1}, S_{2}, S_{3}, \ldots, S_{n}\right\}$. The transmission power levels of the sensor nodes form their set of individual strategy and it is denoted by $P=\left\{P_{0}, P_{1}, P_{2}, P_{3}, \ldots, P_{\max }\right\}$ where $P_{0}$ denotes the inactive state of the nodes, $P_{\max }$ is the maximum transmit power and $P_{1}, P_{2}, P_{3}$ are any power level between $P_{0}$ and $P_{\max }$. The set of fixed strategy profile of all the sensor nodes is denoted by $P_{S}$ where $P_{S}=\left\{P_{S_{1}}, P_{S_{2}}, P_{S_{3}}, \ldots, P_{S_{n}}\right\}$ for sensor nodes $S_{1}, S_{2}, S_{3}$ up to $S_{n}$. All the sensor nodes in the network have their unique power strategy profile, therefore, $P_{0}, P_{1}, P_{2}, P_{3}, \ldots, P_{\text {max }} \in P, P_{S_{1}}, P_{S_{2}}, P_{S_{3}}, \ldots, P_{S_{n}} \in P_{S} \subseteq P$. The sensor nodes within the network are interconnected in a mesh topology and are able to send and receive information from the nodes in their neighborhood. A sensor node requires a minimum SINR to transmit its packet successfully to the receiving node. The SINR for sensor node $S_{1}$ transmitting with $P_{1}$ where $P_{1} \in P_{S_{1}}$ is expressed as:

$$
\operatorname{SINR}_{S_{1}}=\frac{P_{1} G_{1}}{\sum_{S_{2}}^{S_{n}} P_{S \backslash S_{1}} G_{1}+\sigma_{n}},
$$

where $P_{1}$ is the transmission power strategy of sensor node $S_{1}, G_{1}$ denotes the channel gain, $P_{S \backslash S_{1}}$ represents the power of all other transmitting sensor nodes except sensor node $S_{1}$, and $\sigma_{n}$ is the background noise. The work assume that the nodes have omnidirectional antenna thus they can function as a transmitter and receiver, they are able to determine their transmission power, have a unique sensing coverage area, are aware of their channel condition, and the expected SINR of the receiving node which is obtained through periodic acknowledgement.

\section{GAME FORMULATION}

The problem formulated in this paper represents a typical non-cooperative game defined as $G=$ $\left[S,\left\{P_{S}\right\},\left\{U_{S_{1} \in S}\right\}\right]$, where $S$ is the set of the players, $P_{S}$ is the set of their strategy profile, and $U_{S_{1} \in S}$ is the set of their utilities.
Definition 1: Let $P_{1}$ be the least transmit power necessary to achieve the SINR required for a successful packet delivery as defined in (1). $P_{1}$ results in the maximum allowed interference at the neighboring nodes within the cluster. The maximum allowed interference proportion $I$ that the node can tolerate is set to be $\alpha$ and is given for a transmitting sensor node $S_{1}$ as [3]:

$$
\sum_{S_{2}}^{S_{n}} I_{S \backslash S_{1}} \leq \alpha
$$

$I_{S \backslash S_{1}}$ is the allowed interference proportion to all other sensor nodes apart from sensor node $S_{1}$. Any other choice of power strategy of sensor node $S_{1}$ that results in $I_{S \backslash S_{1}}>\alpha$ will cause $S_{1}$ to transmit at a cost which is defined in terms of its transmission power.

Definition 2: The transmitting node transmits at a transmission cost per unit power $C$ at any other power strategy that results in interference proportion $I>\alpha$. The nodes update their power profile continuously to get the optimal transmit power that results in little or no transmission cost, reduced interference and energy consumption. The sensor nodes aim to transmit at this optimal power strategy that maximizes their utility and ensured successful packet delivery.

The expression for the utility of the transmitting sensor node incorporates both the reward and penalty functions which is defined in terms of transmission rate and the cost per unit power incurred. The general utility function for any transmitting sensor node is given as:

$$
U(S, P)=K \log _{2}(1+S I N R)-C(P),
$$

where, $K$ is the utility gain per unit transmission rate of the sensor nodes, $C(P)$ is the transmission cost per unit power of the transmitting node. The cost function $C(P)$ is given in equation (4) as:

$$
C(P)=I P \text {, }
$$

where $I$ is the interference level received by the neighboring nodes from the transmitting node. $I$ is dependent on the transmit power and $P$ is the transmit power of the transmitting node. This cost function is simple and it satisfies the operation characteristics of WSN. Equation (3) is defined for sensor node $S_{1}$ transmitting to node $S_{2}$ as:

$$
\begin{gathered}
U_{S_{1}}\left(S_{1}, P_{1}\right)=K \log _{2}\left(1+\frac{P_{1} G_{1}}{\sum_{S_{2}}^{S_{n}} P_{S \backslash S_{1}} G_{1}+\sigma_{n}}\right)-C P, \\
\forall_{P_{1} \in P_{S_{1}}} .
\end{gathered}
$$

The nodes are not aware of the power strategy of the other nodes, therefore, all the sensor nodes compete 
repeatedly in a non-cooperative manner to maximize their utility by computing the optimization problem in (5):

$$
\begin{aligned}
& \max _{P_{0}<P_{1}<P_{\max }} U_{S_{1}}\left(S_{1}, P_{1}\right), \quad \forall_{S_{1} \in\{S\}}, \\
& \text { s.t } \sum_{S_{2}}^{S_{n}} I_{S \backslash S_{1}} \leq \alpha .
\end{aligned}
$$

\subsection{The Equilibrium Point}

Definition 3: Assume $P_{1}^{*}, \forall_{P_{1}^{*} \in P_{S_{1}} \in P_{S}}$ is a solution to equation (6). A Nash equilibrium exists for the proposed game if $P_{1}^{*}$ is non-empty, convex, and a compact subset of the Euclidean space. Also, if the utility $U_{S_{1}}\left(S_{1}, P_{1}\right)$ is non-zero and continuous in $P_{S_{1}}$ [3] [18].

Proof: Let $P_{1}^{*}$ be the optimal transmission power of sensor node $S_{1}$.

$$
\begin{gathered}
U_{S_{1}}\left(S_{1}, P_{1}\right)=K \log _{2}\left(1+\frac{P_{1} G_{1}}{\sum_{S_{2}}^{S_{n}} P_{S \backslash S_{1}} G_{1}+\sigma_{n}}\right) \\
-C P, \quad \forall_{P_{1} \in P_{S_{1}}} \\
P_{1}^{*}=\arg \max _{P_{1}} K \log _{2}\left(1+\frac{P_{1} G_{1}}{\sum_{S_{2}}^{S_{n}} P_{S \backslash S_{1}} G_{1}+\sigma_{n}}\right) \\
-C P, \quad \forall_{P_{1}^{*} \in P_{S_{1}} \in P_{S}},
\end{gathered}
$$

$P_{1}^{*}$ is the optimal transmission power of sensor node $S_{1}$ and it is within the strategy space $P_{S_{1}}$, therefore, $P_{S_{1}}$ is non-empty, convex and a compact subset of Euclidean space.

$$
P_{0}<P_{1}^{*}<P_{\max } \text { for }\left[P_{0}, P_{1}^{*}, P_{\max } \in P \subseteq P_{S_{1}}\right] \text {, }
$$

The players maximize their given utility for a continuous function seen in (9). Hence from (8),

$$
\begin{gathered}
\frac{\partial U_{S_{1}}\left(S_{1}, P_{1}\right)}{\partial P_{1}}=\frac{K G_{1}}{\ln 2\left(\left(\sum_{S_{2}}^{S_{n}} P_{S \backslash S_{1}} G_{1}+\sigma_{n}\right)+K G_{1} P_{1}\right)}-C P \\
\frac{\partial^{2} U_{S_{1}}\left(S_{1}, P_{1}\right)}{\partial P_{1}^{2}}=-\frac{K G_{1}{ }^{2}}{\ln \left(\left(\sum_{S_{2}}^{S_{n}} P_{S \backslash S_{1}} G_{1}+\sigma_{n}\right)+K G_{1}\right)^{2}}-C P \\
\therefore \quad \frac{\partial^{2} U_{S_{1}}\left(S_{1}, P_{1}\right)}{\partial P_{1}^{2}}<0 .
\end{gathered}
$$

It is seen in (11) and (12) that $U_{S_{1}}\left(S_{1}, P_{1}\right)$ is concave over $P_{S_{1}}$ and thus Nash equilibrium exists in the proposed non-cooperative power control game for WSN where the optimum solution of the game is arg $\max _{P_{1}} U_{S_{1}}\left(S_{1}, P_{1}\right)$ for sensor node $S_{1}$.

\section{SIMULATION RESULTS AND DISCUSSION}

The performance of the proposed scheme was evaluated by computing the interference proportion, utility, transmission rate, transmission cost, and SINR of the sensor nodes. The simulation set up consist 10 evenly distributed sensor nodes per cluster and each node can either act as a receiver or as a transmitter. For ease of computation, the nodes were modeled in an area of $150 \times 150 \mathrm{~m}^{2}$ and at the coordinate of 50,50 to the sink node.

Table 1: Simulation Parameters, [3, 19].

\begin{tabular}{cc}
\hline Parameters & Values \\
\hline Bandwidth & $1 \mathrm{MHZ}$ \\
Noise Power & $5 \times 10^{-15} \mathrm{~W}$ \\
Channel Gain & $7.75 \times 10^{-13} / d_{i}^{3.6}$ \\
Maximum Power & $100 \mathrm{~W}$ \\
Minimum Power & $0 \mathrm{~W}$ \\
\hline
\end{tabular}

The nodes transmit within their coverage area, their power level is $0 W$ during inactive state while the maximum allowable transmit power is $100 \mathrm{~W}$. The simulation parameter is shown in table 1 . The interference level at the receiving nodes and the resulting incurred transmission cost of the transmitting nodes were investigated at different transmit power for $P=\left\{P_{0}, P_{1}, P_{2}, P_{3}, \ldots, P_{\max }\right\} \subseteq P_{S}$. The effect of the proposed scheme on the transmission cost and SINR versus the transmission power was shown in Figure 2 and Figure 3 respectively. It was observed in Figure 2, that the transmission cost increases with increase in transmit power. Though the increase in power level ensured successful packet delivery, it does not result in efficient network resource utilization because the sensor nodes used up their energy very fast. Under the proposed scheme, the interference proportion was seen to be almost negligible before it increased exponentially. This exponential increase results in very low payoff values as seen in Figure 4.

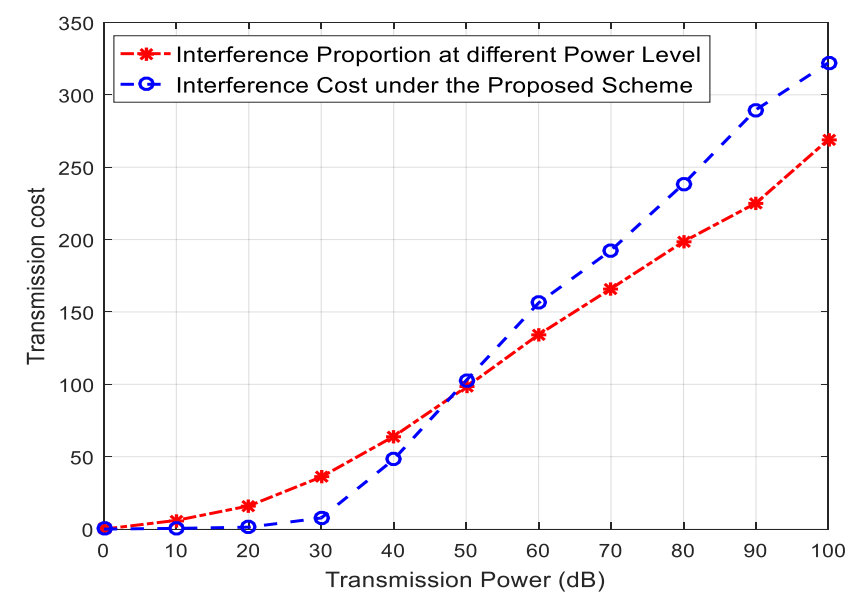

Figure 2: Relationship between Transmission Cost and Transmission Power

Vol. 38, No. 2, July 2019 
The sensor nodes achieved an SINR value required to deliver their packet to the receiving nodes at the optimal transmit power as seen in Figure 3. The interference proportion is measured in terms of the interference level received by the other nodes and it is dependent on transmit power. This discussion is in accordance with section 3 and it showed that, though the nodes achieved a higher SINR, but the SINR values did not result in a good utility value. The sensor nodes aim to transmit at the optimal power level that maximizes their utility and result in good transmission sum rate. The utility of the sensor nodes was investigated at different transmit power in Figure 4 by computing the transmission rate of the sensor nodes at optimal power and the transmission cost defined in terms of the interference proportion during transmission. The utility value increased gradually until it reaches the optimal point at $P_{1}^{*}$ after it began to reduce exponentially. This shows that at $P_{1}>0 \leq$ $P_{1}^{*}$, the transmission of the nodes results in a tolerable interference proportion and they incurred an almost negligible cost. At $P_{1}>P_{1}^{*}$, the sensor nodes have low utility value and this further justifies the defined transmission cost which enforces a degree of cooperation between the sensor nodes.

The transmission sum rate of the sensor nodes was evaluated under the interference power threshold and under different power level without the constraint as shown in Figure 5. It was observed that all the nodes have a higher transmission sum rate when they transmit at the optimal power level except for node six which was located at the farthest distance to the sink node. This is contrary to what was seen when the transmission cost was not imposed. The sum rates of the nodes that transmitted higher were greatly reduced because they incurred increase interference level.

To further validate the performance of the work presented in this paper, the proposed model is compared with the scheme presented in the work of [13] where sensor nodes chose their transmit power independently to achieve a target SINR at the master sensor. The Pareto Optimality of their work was not verified and the equilibrium point is restricted and distance dependent. In our formulated power control scheme, the optimal power of all the sensor nodes was determined by considering all the resulting interference from all the transmitting nodes. We introduced a power constrain that results in the optimal power strategy of all the nodes and it results in an increased utility value, efficient energy usage, and reduced transmission cost when compared to the work presented in [13].

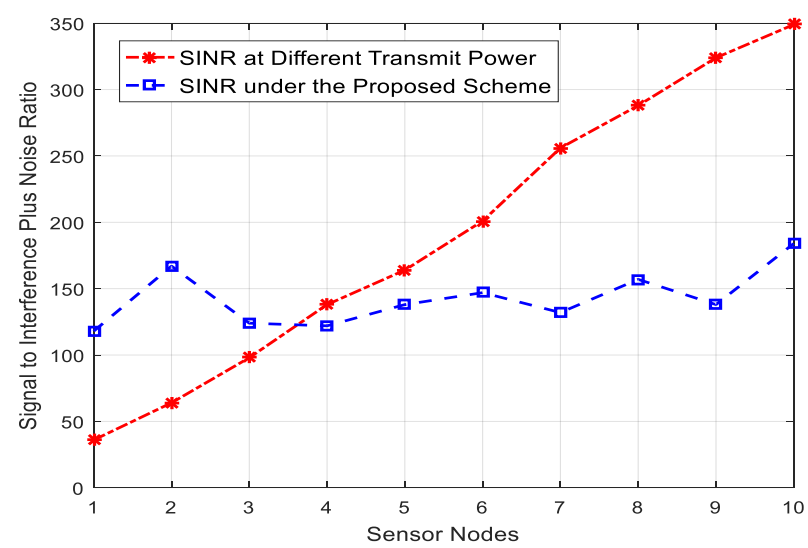

Figure 3: Signal to Interference Ratio at different Transmission Power.

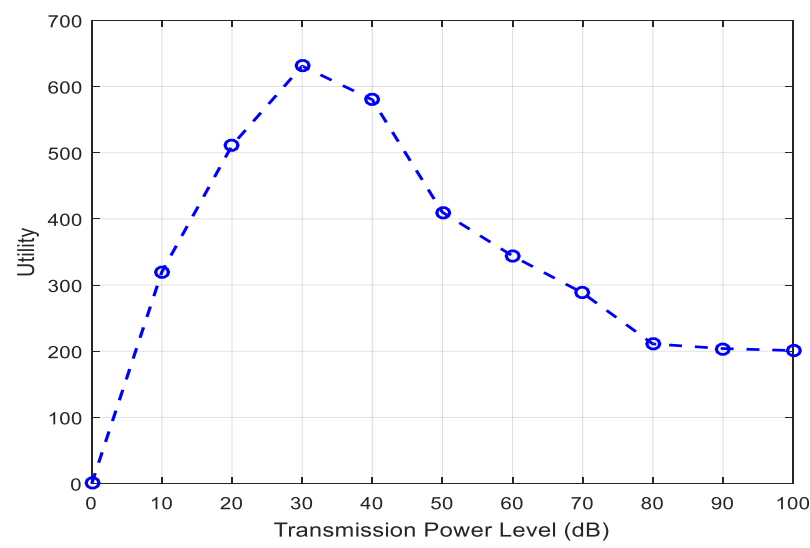

Figure 4: Utility at different Transmission Power under the Interference Constraint

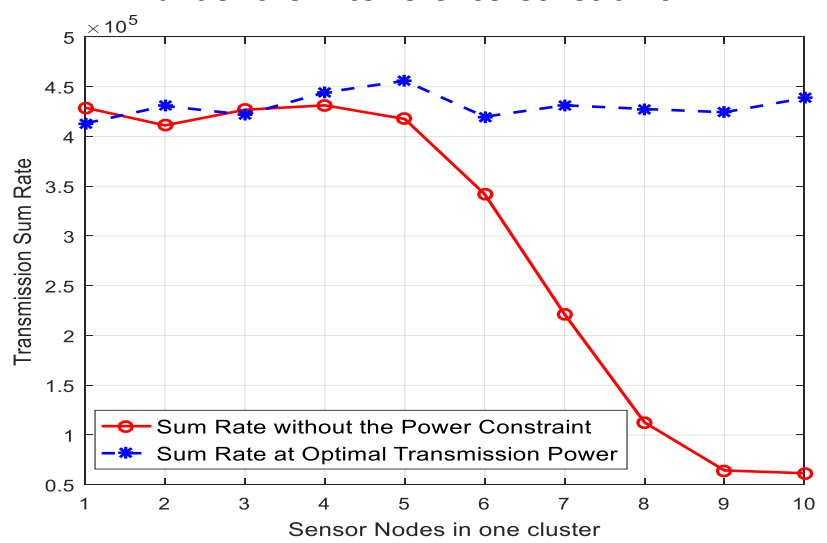

Figure 5: Transmission Sum Rate of different Sensor Nodes in a Cluster

\section{CONCLUSION}

In this paper, a power control game model that reduces interference and energy consumption in a wireless sensor network is presented. A noncooperative power control game was formulated among the sensor nodes in the modeled network by setting a transmission power threshold at the receiving nodes which ensures the optimal transmission of all 
the nodes. The utility function that incorporates both the profit and the cost function which ensures that all the sensor nodes transmit at their optimal power was defined and the existence of the Nash equilibrium in the proposed game was determined. The performance of the proposed scheme was evaluated and it results in increased transmission sum rate, reduced interference within the network, and increased lifespan of the sensor nodes. The work carried out in this paper can be extended to the other technical challenges in wireless sensor network which include routing issues, effective sensor mobility, and sensor security.

\section{REFERENCES}

[1] S. Sengupta, M. Chatterjee, and K.A. Kwiat. "Game Theoretic Framework for Power Control in Wireless Sensor Networks" IEEE transactions on computers, Vol. 59, No. 2. pp. 231 - 242, February, 2010.

[2] V.E Ekong and U.D Ekong "A survey of security vulnerabilities in wireless sensor network", Nigerian Journal of Technology, Vol. 35, No. 2 pp. $392-397,2016$.

[3] Ladipo, O.I and Gbenga-Ilori A.O. "A NonCooperative Power Control Game for Wireless Sensor Networks", Proceeding of $13^{\text {th }}$ UNILAG Annual Conference \& Fair - An International Conference of the Sciences and Humanities. August 28 - 30, 2018, University of Lagos, Akoka - Lagos.

[4] H-Y. Shi, W-L. Wang, N-M. Kwok, and S-Y. Chen, "Game theory for wireless sensor networks", $A$ survey Sensors - MPDI Journals, pp. 9055 - 9097, 2012.

[5] T. Alpcan, T. Basar, and S. A. Dey, "Power control game based on outage probabilities for multi-cell wireless data networks. In Proceedings of the 2004 American Control Conference, Boston, MA, 30 June-2 July, 2004.

[6] F. Tsuo, H. Tan, Y. Chew, and H. Wei, "Energyaware transmission control for wireless sensor networks powered by ambient energy harvesting: A game-theoretic approach." In Proceedings of IEEE International Conference on Communications, Kyoto, Japan, June, 2011.

[7] V. Krishnamurthy, "Decentralized activation in dense sensor networks via global games". IEEE Transaction on Signal. Processing. 56, pp. 4936 4950, 2008.

[8] Z. Han, D. Niyato, W. Saad, T. Basar, and A. Hjørungnes, "Game Theory in Wireless and
Communication Networks Theory, Models, and Applications." Cambridge University press, 2012.

[9] I.F. Akyildiz, W. Su, Y. Sankarasubramaniam, E. Cayirci "A survey on sensor networks", IEEE Communications Magazine. Vol. 40, Issue 8, August, 2002.

[10] J. Juncheng, and Z. Qian, "A non-cooperative power control game for secondary spectrum sharing". IEEE Communications Society ICCC, 2007.

[11] D. Yang, J. Zhang, X. Fang, A. Richa, and G. Xue, "Optimal transmission power control in the presence of a smart jammer". GLOBECOM Wireless Networking Symposium, pp. 5506 - 5511, 2012.

[12] H. Lee, H. Kwon, A. Motskin, L. Guibas, "Interference aware MAC protocol for wireless networks by a game theoretic approach". In Proceeding of IEEE INFOCOM, 2009.

[13] W. Tushar, D. Smith, T. A. Lamahewa, and J. A. Zang "Non-cooperative Power Control Game in Multi-Source Wireless Sensor Network", Australian Communications Theory Workshop (AusCTW), pp. 43-48, 2012.

[14] G. Yang, and G. Zhang, "A power control algorithm based on non-cooperative game for wireless sensor networks", In Proceedings of 2011 International Conference on Electronic \& Mechanical Engineering and Information Technology, Harbin, China, 12-14, August, 2011.

[15] J. Huang, R.A. Berry and M.L. Honig, "Distributed interference compensation for wireless networks." IEEE Journal of Selected Area in Communication. Vol. 24, pp. 1074-1084, 2006.

[16] B.C. Chen, C.L Na, and T.X. Zhou, T. "Convergence and fairness of power control algorithm for wireless sensor networks." Journal of Harbin Institute of Technology, Vol. 40, pp. 475-478, 2008.

[17] C. Na, D. Lu, T. Zhou, and L. Li, "Distributed power control algorithm based on game theory for wireless sensor networks" Elsevier Journal of Systems Engineering and Electronics, Vol. 18, No. 3, pp. 622-627, 2007.

[18] X. Xu, Y. Li, R. Gao, and X. Tao, "Joint Voronoi diagram and game theory-based power control scheme for the HetNet small cell networks". EURASIP Journal on Wireless Communications and Networking 2014, 2014:213.

[19] Z. Gengzhong, L. Sanyang, and Q. Xiaogang, "A power control algorithm based on non-cooperative game for wireless CDMA sensor networks". International Journal of Digital Content Technology and its applications Vol. 4, No 3, June, 2010. 